> L'autophagie est un mécanisme clé du maintien del'homéostasie cellulaire et de l'organisme. Par conséquent, des défauts dans les composants essentiels de la machinerie autophagique sont associés étiologiquement à une variété de pathologies humaines dont des troubles infectieux, des maladies cardiovasculaires, des maladies neurodégénératives et le cancer. Plusieurs manipulations qui augmentent la durée de vie des organismes modèles en laboratoire, comme la restriction calorique, font intervenir l'autophagie. Nous discutons dans cette revue la relation intime qui existe entre la préservation de la forme des cellules et de l'organisme par l'autophagie et le vieillissement en bonne santé. <

La macroautophagie (que nous appellerons « autophagie » tout au long du texte) est un processus cellulaire par lequel du matériel cytoplasmique d'origine endogène ou exogène est initialement séquestré par des organites à double membranes nouvellement formés (communément connus sous le nom d'autophagosomes) et éventuellement dégradés par les lysosomes [1] (Figure 1). Dans des conditions physiologiques, la dégradation autophagique se produit à un niveau basal, assurant l'élimination de matériels potentiellement dangereux qui peuvent s'accumuler, du fait du fonctionnement cellulaire normal (par exemple, les mitochondries perméabilisées, des agrégats de protéines redox-actives $^{1}$ ) [2]. Ainsi, l'autophagie fonctionnet-elle sans relâche comme un système de contrôle de qualité pour le maintien de l'homéostasie cellulaire.

L'autophagie intervient également au croisement de la quasi-totalité des réponses cellulaires au stress [3]. En effet, le flux autophagique peut être finement adapté à de multiples altérations du microenvironnement, à la fois intracellulaire et extracellulaire, dont des stress nutritionnels, métaboliques, physiques et chimiques [4]. Dans

${ }^{1}$ Des agrégats qui favorisent la formation d'espèces réactives de l'oxygène.

\section{Autophagie, bonne santé et longévité}

\author{
Lorenzo Galluzzi ${ }^{1-6}$, \\ José Manuel Bravo-San Pedro ${ }^{2-6}$, \\ Guido Kroemer ${ }^{2-5,7-9}$ \\ Traduction française de Thierry Jouault
}

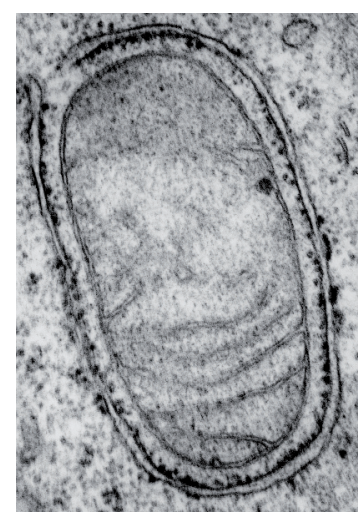

${ }^{1}$ Department of radiation oncology, Weill Cornell Medical College, New York, Ny, ÉtatsUnis ;

${ }^{2}$ Inserm UMRS 1138, Paris, France ; ${ }^{3}$ Université Paris Descartes/ Paris V, Sorbonne Paris Cité, Paris, France ;

${ }^{4}$ Université Pierre et Marie Curie/ Paris VI, Paris, France ;

${ }^{5}$ Équipe 11 labellisée par la Ligue nationale contre le cancer, Centre de recherche des Cordeliers, Paris, France ;

ce contexte, l'autophagie est généralement à l'origine de fonctions de cytoprotection, comme en témoigne le fait que des cellules soumises à l'inhibition pharmacologique ou génétique de l'autophagie sont plus susceptibles de succomber à un stress que leurs homologues compétentes [3]. En plus de préserver le microenvironnement intracellulaire de la dégénérescence physiologique ou pathologique, l'autophagie supporte d'importantes fonctions homéostatiques extracellulaires qui se manifestent au niveau de l'organisme $[5,6]$.

Les réponses autophagiques peuvent fonctionner selon deux modes qui sont distincts mais pas mutuellement exclusifs [7]. D'une part, les autophagosomes peuvent séquestrer de manière relativement non sélective un matériel cytoplasmique qui n'est pas essentiel pour la survie cellulaire. Une telle réponse a principalement des objectifs nutritionnels ou métaboliques permettant aux cellules de survivre en cas de carence en nutriments en recyclant le matériel cytoplasmique disponible [8]. Les autophagosomes peuvent, d'autre part, recueillir des composants cellulaires de manière hautement spécifique. Dans ce cas, la machinerie autophagique intervient face à une entité potentiellement nocive qui s'accumule en réponse à une situation spécifique [7]. Par exemple, les cellules exposées à des toxines mitochondriales réagissent en activant une réponse autophagique très sélective, appelée «mitophagie », ciblant spécifiquement les mitochondries perméabilisées (Figure 1) $[9,53](\rightarrow)$.

$(\rightarrow)$ Voir la Synthèse de $P$. Vigié et N. Camougrand, page 231 de ce numéro 


\begin{tabular}{|c|c|c|c|}
\hline Intervention & Organisme modèle & Lien avec l'autophagie & Référence \\
\hline \multicolumn{4}{|l|}{ Nutritionnelle } \\
\hline \multirow{3}{*}{ Restriction calorique } & Saccharomyces cerevisiae & Établi par délétion de MAFI & [22] \\
\hline & Macaque rhésus (macaca mulatta) & Pas établi directement & [38] \\
\hline & Mus musculus & Établi dans les souris Sirtl $1^{+/-}$ & [33] \\
\hline \multicolumn{4}{|l|}{ Pharmacologique } \\
\hline \multirow{2}{*}{ Rapamycine } & Drosophila melanogaster & Établi par délétion de Atg5 & {$[30]$} \\
\hline & Mus musculus & Pas établi directement & {$[34,35]$} \\
\hline \multirow{3}{*}{ Resveratrol } & Saccharomyces cerevisiae & Pas établi directement & [23] \\
\hline & Caenorhabditis elegans & Établi par délétion de bec-l & {$[25]$} \\
\hline & Mus musculus & Pas établi directement & {$[36]$} \\
\hline \multirow{3}{*}{ Spermidine } & Levure By4741 & Établi par délétion de ATG7 & {$[24]$} \\
\hline & Caenorhabditis elegans & Établi par délétion de bec-l & [24] \\
\hline & Drosophila melanogaster & Établi par délétion de Atg7 & [24] \\
\hline \multicolumn{4}{|l|}{ Génétique } \\
\hline Suppression de TORI & Saccharomyces cerevisiae & Pas établi directement & [22] \\
\hline $\begin{array}{l}\text { Mutation de daf-2, eat-2 } \\
\text { ou cnb-1 }\end{array}$ & Caenorhabditis elegans & Établi par suppression de bec-1, atg-7, unc-51 ou lgg-1 & {$[26-28]$} \\
\hline Surexpression de $h l h-30$ & Caenorhabditis elegans & Établi par suppression de atg-18 & [29] \\
\hline $\begin{array}{l}\text { Surexpression de Atg8a ou } \\
\text { Foxo }\end{array}$ & Drosophila melanogaster & Pas établi directement & {$[31,32]$} \\
\hline Surexpression de Atg5 & Mus musculus & Pas établi directement & [37] \\
\hline
\end{tabular}

Tableau I. Exemples d'activateurs autophagiques augmentant la durée de vie. Atg : autophagy related; bec-1:Beclin homolog 1 ; cnb-1:calcineurin $b-1$; daf-2 : insulin-like receptor subunit beta-2 ; eat-2 : neuronal acetylcholine receptor subunit eat-2 ; Foxo : Forkhead box, 0 ; hlh-30: helix loop helix 30 ; MAFI : RNA polymerase III-inhibiting protein MAF1; Sirt 1 : sirtuin 1; TOR1 : phosphatidylinositol kinase-related protein kinase ; unc-51: serine/threonine-protein kinase unc-51.

Le rôle important des réponses autophagiques dans le maintien de l'homéostasie (cellulaire et de l'organisme) a été montré par les associations entre les défauts de la machinerie moléculaire impliquée dans l'autophagie et l'étiologie de multiples pathologies cliniquement pertinentes, dont (sans être exhaustif) les maladies infectieuses, les maladies cardiovasculaires, les maladies neurodégénératives et le cancer [10-12]. La durée de vie en bonne santé de multiples organismes modèles (la levure Saccharomyces cerevisiae [13-15], le ver Caenorhabditis elegans [16-20], la mouche Drosophila melanogaster [15, 21-23], la souris Mus musculus [24-28], et le singe Macaca mulatta (macaque rhésus) [29]) peut être prolongée expérimentalement par des conditions qui activent l'autophagie (Tableau I), avec la nécessité, quasi-invariablement, d'une machinerie autophagique intacte [30]. Nous examinerons dans cette revue les relations existant entre capacité de l'autophagie à préserver l'intégrité cellulaire et celle de l'organisme et le vieillissement en bonne santé.

\section{Homéostasie intracellulaire et vieillissement}

Parmi d'autres altérations, le vieillissement s'accompagne de l'accumulation de dommages macromoléculaires touchant tous les compartiments sous-cellulaires, y compris le noyau, qui conduisent finalement à l'une ou l'autre des conséquences délétères: la sénescence cellulaire ou la mort cellulaire régulée (ou RCD pour regulated cell death) [31]. À noter que la sénescence cellulaire et la RCD affectent également le compartiment des cellules souches qui vieillissent 


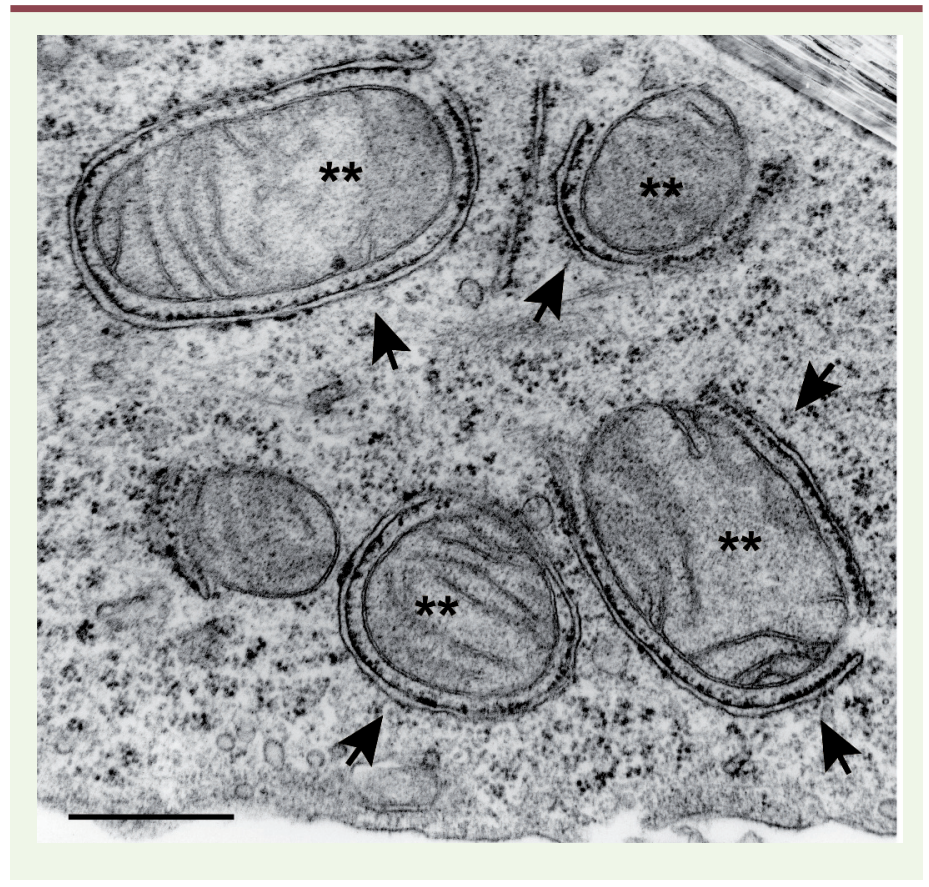

Figure 1. Photo d'autophagosomes au microscope électronique. Cellule de cancer bronchique non à petites cellules (lignée humaine H1975) contenant plusieurs autophagosomes à différentes étapes de maturation (flèches) autour de mitochondries endommagées $(* *)$. Échelle $=500 \mathrm{~nm}$.

[32], ce qui explique que le vieillissement soit associé à une dégénérescence structurelle et fonctionnelle progressive de plusieurs tissus et organes, qui n'est plus compensée par les cellules souches [31]. Ainsi, le vieillissement des tissus et des organes conduit à la perte des cellules fonctionnelles via la RCD, et à l'accumulation de cellules sénescentes qui sont vivantes mais (1) qui ont cessé leurs activités physiologiques, et (2) qui sécrètent de nombreux médiateurs bioactifs dont certains favorisent la sénescence des cellules voisines, comme les espèces réactives de l'oxygène (ROS) [32]. Dans certains tissus, comme le système nerveux central (SNC) et le muscle squelettique, le vieillissement peut également s'accompagner d'un dysfonctionnement mitochondrial progressif aboutissant à une inefficacité bioénergétique chronique et/ou du dépôt d'agrégats cytotoxiques et/ou de protéines redox-actives [11,33].

L'autophagie ralentit le vieillissement de l'organisme en particulier en limitant l'accumulation des dommages macromoléculaires qui entraînent la sénescence cellulaire et la RCD. Ainsi, des réponses autophagiques adéquates sont essentielles au maintien d'un réseau mitochondrial efficacement couplé et pour l'élimination des agrégats protéiques [2, 34]. Ceci est d'une extrême importance dans le contexte du vieillissement pour au moins deux raisons: (1) les mitochondries découplées sont une source majeure de ROS intracellulaires qui endommagent directement I'ADN et la membrane lipidique, promouvant la sénescence cellulaire ou la $\operatorname{RCD}[32,35]$; et (2) le contrôle de qualité du réseau mitochondrial, réalisé par l'autophagie, permet de préserver une efficacité bioénergétique optimale (en particulier dans les tissus ayant des exigences de haute énergie comme le SNC) [36]. L'autophagie s'oppose également à la sénescence et à la RCD en favorisant directement la réparation d'ADN altéré [37], en veillant à la dégradation des fragments d'hétérochromatine endommagée au-delà des capacités de réparation [38], et en éliminant d'autres structures potentiellement génotoxiques, comme les micronoyaux qui apparaissent lors de divisions cellulaires asymétriques et les rétrotransposons [39]. Enfin, l'autophagie protège les cellules contre les infections virales et bactériennes potentiellement létales [40]. Elle permet ainsi de limiter la perte de tissu, lorsqu'un compartiment de cellules souches est altéré comme chez les personnes âgées. Les fonctions cytoprotectrices de l'autophagie interfèrent également avec le processus de transformation maligne [10], le vieillissement étant lui-même associé à une incidence accrue de plusieurs tumeurs. La capacité des réponses autophagiques à préserver l'homéostasie intracellulaire bénéficie également aux compartiments de cellules souches [41], ce qui a un impact positif majeur sur la préservation de l'architecture tissulaire au cours du vieillissement. Par sa capacité à préserver l'intégrité des cellules parenchymateuses et des cellules souches, l'autophagie permet donc (au moins en partie) de ralentir le vieillissement.

\section{Homéostasie de l'organisme et vieillissement}

Le vieillissement s'accompagne non seulement de modifications touchant intrinsèquement les cellules, mais également d'une multitude d'altérations affectant l'ensemble de l'organisme. Le vieillissement est associé à (et peut-être favorisé par) des changements au niveau de la constitution du système immunitaire et de son activité entraînant souvent une inflammation systémique de faible intensité (appelée en anglais inflammaging) [42]. Des données épidémiologiques indiquent en parallèle une forte association entre l'âge (> 65 ans) et la détérioration de divers circuits neuroendocriniens à l'origine de maladies métaboliques comme l'obésité, le diabète, la résistance à l'insuline et le syndrome métabolique [43]. De même, l'incidence de plusieurs tumeurs malignes augmente considérablement avec l'âge. Cela ne reflète pas seulement la propension accrue des cellules en vieillissement pour la transformation maligne (voir ci-dessus), mais cela implique également des mécanismes extracellulaires [44]. Il est en effet maintenant bien établi que les tumeurs apparaissent dans le contexte d'une relation intime, avec plusieurs facettes et souvent bidirectionnelle, avec le système immunitaire; l'évolution clinique 


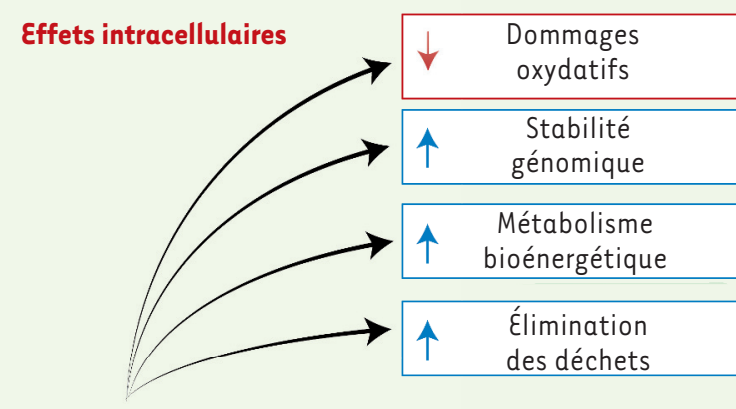

AUTOPHAGIE

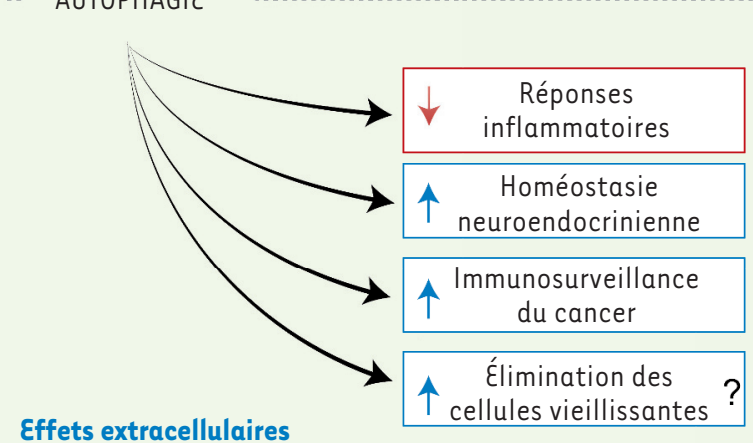

Figure 2. Liens entre l'autophagie et le vieillissement en bonne santé. Des réponses autophagiques efficaces permettent de limiter l'accumulation de matériel cytoplasmique potentiellement nocif comme les mitochondries perméabilisées ou les agrégats de protéines redox-actives, limitant de facto des dommages oxydatifs aux macromolécules et les instabilités génomiques, tout en optimisant le métabolisme. Au niveau de l'organisme, l'autophagie a de nombreux effets bénéfiques : elle limite les réponses inflammatoires, entretient les capacités neuroendocriniennes de l'ensemble de l'organisme, et maintient l'immunosurveillance vis-à-vis des cellules (pré)malignes et (selon certains auteurs) des cellules vieillissantes. Finalement, ces fonctions de protection (et peut-être d'autres) fournissent à l'autophagie la capacité de promouvoir le vieillissement sans pathologies et d'augmenter la durée de vie, ceci dans plusieurs organismes modèles. ne se manifestant que lorsqu'elles échappent à l'immunosurveillance anticancéreuse [45].

Au moins une partie des effets antivieillissement de l'autophagie repose sur sa capacité à neutraliser chacun des effets extracellulaires du processus de vieillissement. Chez les mammifères, les réponses autophagiques des macrophages limitent la mise en place de l'inflammation systémique en réduisant la production de médiateurs proinflammatoires, comme l'interleukine 1 bêta $(I L-1 \beta)$ et l'interleukine 18 (IL-18) [46]. La machinerie autophagique est par ailleurs nécessaire pour transmettre les effets bénéfiques de l'exercice physique au métabolisme du glucose et à la sensibilité à l'insuline [47]. En effet, l'autophagie permet de préserver les circuits neuroendocriniens généralement détériorés au cours du vieillissement. Elle a également été impliquée dans plusieurs étapes des réponses immunitaires innées et adaptatives [6, 48], ainsi que dans la libération des signaux immunostimulants par des cellules cancéreuses qui meurent en réponse à certains types de traitement [49].

\section{Conclusion}

L'autophagie est un mécanisme très conservé chez les eucaryotes. Elle participe à des fonctions homéostatiques qui sont critiques dans des organismes aussi éloignés que la levure ou l'homme. Curieusement, au cours de l'évolution, l'autophagie a considérablement élargi son champ d'action, bien au-delà de la limite physique que représente la membrane plasmique de la cellule. Ainsi, chez les mammifères modernes elle supporte des effets au sein des cellules elles-mêmes, qui leur sont bénéfiques, mais également au niveau extracellulaire, dans le milieu extérieur, soutenant en cela son importante implication dans le ralentissement du vieillissement (au moins dans un cadre expérimental) (Figure 2).

Dans les situations dans lesquelles les deux effets (intra- et extracellulaire) apparaissent en opposition et se neutralisent l'un l'autre, ce sont les effets extracellulaires qui prédominent. Ainsi, dans les cellules cancéreuses exposées à des immunothérapies, l'activation de l'autophagie est supposée promouvoir simultanément la survie des cellules et l'activation de la réponse immunitaire anticancéreuse [10]. Les manipulations génétiques qui conduisent à l'inhibition de l'autophagie, comme la suppression permanente du gène Atg5 qui code l'une des protéines ATG (autophagy related protein), limite (plutôt qu'améliore) les réponses thérapeutiques chez des animaux immunocompétents [50]. Chez les animaux immunodéficients, en revanche, la suppression d'Atg5 sensibilise les cellules cancéreuses au traitement ${ }^{2}$ [50]. L'autophagie semble être un processus important qui est impliqué

${ }^{2}$ Dans un modèle dans lequel les conséquences immunologiques extracellulaires des réponses autophagiques ne peuvent pas être appréciées. 
dans la préservation de l'intégrité physique (en anglais, fitness), qui repose sur un mécanisme à la fois intra- et extracellulaire. Ainsi, les activateurs pharmacologiques de l'autophagie, tels des agents qui simulent la restriction calorique [5l], semblent présenter une utilité non seulement pour le traitement de plusieurs pathologies ayant pour origine des défauts autophagiques [52], mais également dans le cadre plus large de l'allongement de la longévité. $\diamond$

\section{SUMMARY}

\section{Autophagy, fitness and longevity}

Autophagy is a key mechanism for the maintenance of intracellular and organismal homeostasis. Accordingly, defects in core components of the autophagic machinery are etiologically associated with a variety of human pathologies, including infectious disorders, cardiovascular diseases, neurodegenerative conditions, and cancer. Intriguingly, several maneuvers that increase the lifespan of model organisms in the laboratory, like caloric restriction, do so in an autophagy-dependent manner. Here, we briefly discuss the intimate relationship between the autophagic preservation of intracellular and organismal fitness and healthy aging. $\diamond$

\section{REMERCIEMENTS}

Les auteurs sont soutenus par la Ligue contre le Cancer (équipe labellisée); l'Agence national de la recherche (ANR) - Projets Blancs; l'ANR dans le cadre de E-Rare-2 (ERA-Net for research on rare diseases); l'Association pour la recherche sur le cancer (ARC) ; le Cancéropôle lle-de-France; l'Institut National du Cancer (INCa); la Fondation Bettencourt Schueller; la Fondation de France; la Fondation pour la Recherche Médicale (FRM); la commission européenne (ArtForce); l'European Research Council (ERC); le LabEx Immuno-Oncology; le SIRIC (sites de recherche intégrée sur le cancer) Stratified Oncology Cell DNA Repair and Tumor Immune Elimination (SOCRATE); the SIRIC Cancer Research and Personalized Medicine (CARPEM); la fondation suisse Bridge; L'Institut suisse pour la recherche expérimentale sur le cancer (ISREC); Paris Alliance of Cancer Research Institutes (PACRI).

\section{LIENS D'INTÉRÊT}

Les auteurs déclarent n'avoir aucun lien d'intérêt concernant les données publiées dans cet article.

\section{RÉFÉRENCES}

1. Lamb CA, Yoshimori T, Tooze SA. The autophagosome: origins unknown, biogenesis complex. Nat Rev Mol Cell Biol $2013 ; 14: 759-74$.

2. Green DR, Galluzzi L, Kroemer G. Mitochondria and the autophagy-inflammation-cell death axis in organismal aging. Science $2011 ; 333: 1109-12$.

3. Kroemer G, Marino G, Levine B. Autophagy and the integrated stress response. Mol cell $2010 ; 40$ : 280-93.

4. Kaur J, Debnath J. Autophagy at the crossroads of catabolism and anabolism. Nat Rev Mol Cell Biol $2015 ; 16: 461-72$.

5. Tsakiri EN, Trougakos IP. The amazing ubiquitin-proteasome system: structural components and implication in aging. Int Rev Cell Mol Biol $2015 ; 314$ : 171-237.

6. Ma Y, Galluzzi L, Zitvogel L, Kroemer G. Autophagy and cellular immune responses. Immunity $2013 ; 39: 211-27$.

7. Sica V, Galluzzi L, Bravo-San Pedro JM, et al. Organelle-specific initiation of autophagy. Mol cell $2015 ; 59: 522-39$.

8. Galluzzi L, Pietrocola F, Levine B, Kroemer G. Metabolic control of autophagy. Cell 2014 ; 159 : 1263-76.

9. Quiros PM, Langer T, Lopez-Otin C. New roles for mitochondrial proteases in health, ageing and disease. Nat Rev Mol Cell Biol $2015 ; 16: 345-59$.

10. Galluzzi L, Pietrocola F, Bravo-San Pedro JM, et al. Autophagy in malignant transformation and cancer progression. EMBO J $2015 ; 34: 856-80$.

11. Menzies FM, Fleming A, Rubinsztein DC. Compromised autophagy and neurodegenerative diseases. Nat Rev Neurosci $2015 ; 16: 345-57$.
12. Shirakabe A, Ikeda Y, Sciarretta S, et al. Aging and autophagy in the heart. Circ Res $2016 ; 118: 1563-76$.

13. Cai $Y$, Wei $Y H$. Stress resistance and lifespan are increased in C. elegans but decreased in S. cerevisiae by mafr-1/mafl deletion. Oncotarget $2016 ; 7$ : 10812-26.

14. Howitz KT, Bitterman KJ, Cohen HY, et al. Small molecule activators of sirtuins extend Saccharomyces cerevisiae lifespan. Nature $2003 ; 425$ : 191-6.

15. Eisenberg $\mathrm{T}$, Knauer $\mathrm{H}$, Schauer $\mathrm{A}$, et al. Induction of autophagy by spermidine promotes longevity. Nat Cell Biol 2009 ; 11 : 1305-14.

16. Morselli $\varepsilon$, Maiuri MC, Markaki M, et al. Caloric restriction and resveratrol promote longevity through the Sirtuin-1-dependent induction of autophagy. Cell Death Dis 2010 ; 1 : el0.

17. Melendez A, Talloczy Z, Seaman M, et al. Autophagy genes are essential for dauer development and life-span extension in C. elegans. Science 2003; $301: 1387-91$.

18. Jia K, Levine B. Autophagy is required for dietary restriction-mediated life span extension in C. elegans. Autophagy 2007 ; 3 : 597-9.

19. Dwivedi M, Song HO, Ahnn J. Autophagy genes mediate the effect of calcineurin on life span in C. elegans. Autophagy $2009 ; 5: 604-7$.

20. Lapierre LR, De Magalhaes Filho CD, McQuary PR, et al. The TFEB orthologue $\mathrm{HLH}-30$ regulates autophagy and modulates longevity in Caenorhabditis elegans. Nat Commun $2013 ; 4: 2267$.

21. Bjedov I, Toivonen JM, Kerr F, et al. Mechanisms of life span extension by rapamycin in the fruit fly Drosophila melanogaster. Cell metabolism 2010 ; $11: 35-46$

22. Simonsen A, Cumming RC, Brech A, et al. Promoting basal levels of autophagy in the nervous system enhances longevity and oxidant resistance in adult Drosophila. Autophagy 2008; 4 : 176-84.

23. Demontis F, Perrimon N. FOXO/4E-BP signaling in Drosophila muscles regulates organism-wide proteostasis during aging. Cell $2010 ; 143: 813-25$.

24. Mercken EM, Hu J, Krzysik-Walker S, et al. SIRTl but not its increased expression is essential for lifespan extension in caloric-restricted mice. Aging Cell 2014 ; 13 : 193-6.

25. Harrison DE, Strong R, Sharp ZD, et al. Rapamycin fed late in life extends lifespan in genetically heterogeneous mice. Nature $2009 ; 460$ : 392-5.

26. Miller RA, Harrison DE, Astle CM, et al. Rapamycin-mediated lifespan increase in mice is dose and sex dependent and metabolically distinct from dietary restriction. Aging Cell $2014 ; 13: 468-77$.

27. Baur JA, Pearson KJ, Price NL, et al. Resveratrol improves health and survival of mice on a high-calorie diet. Nature $2006 ; 444: 337-42$.

28. Pyo J0, Yoo SM, Ahn HH, et al. Overexpression of Atg5 in mice activates autophagy and extends lifespan. Nat Commun $2013 ; 4: 2300$.

29. Colman RJ, Beasley TM, Kemnitz JW, et al. Caloric restriction reduces agerelated and all-cause mortality in rhesus monkeys. Nat Commun $2014 ; 5$ : 3557.

30. Madeo F, Zimmermann A, Maiuri MC, Kroemer G. Essential role for autophagy in life span extension. J Clin Invest $2015 ; 125: 85-93$.

31. Lopez-Otin C, Blasco MA, Partridge L, et al. The hallmarks of aging. Cell $2013 ; 153: 1194-217$.

32. Munoz-Espin D, Serrano M. Cellular senescence: from physiology to pathology. Nat Rev Mol Cell Biol 2014 ; 15 : 482-96.

33. Knott AB, Perkins G, Schwarzenbacher R, Bossy-Wetzel $\varepsilon$. Mitochondrial fragmentation in neurodegeneration. Nat Rev Neurosci 2008 ; 9 : 505-18.

34. Wong $\varepsilon$, Bejarano $\varepsilon$, Rakshit $M$, et al. Molecular determinants of selective clearance of protein inclusions by autophagy. Nat Commun $2012 ; 3: 1240$.

35. Fang EF, Scheibye-Knudsen M, Chua KF, et al. Nuclear DNA damage signalling to mitochondria in ageing. Nat Rev Mol Cell Biol 2016; 17 : 308-21.

36. Burte F, Carelli V, Chinnery PF, Yu-Wai-Man P. Disturbed mitochondrial dynamics and neurodegenerative disorders. Nat Rev Neurol 2015 ; 11 : 11-24.

37. Karantza-Wadsworth V, Patel S, Kravchuk 0, et al. Autophagy mitigates metabolic stress and genome damage in mammary tumorigenesis. Genes Dev $2007 ; 21: 1621-35$.

38. Dou Z, Xu C, Donahue G, et al. Autophagy mediates degradation of nuclear lamina. Nature 2015 ; 527 : 105-9.

39. Guo H, Chitiprolu M, Gagnon D, et al. Autophagy supports genomic stability by degrading retrotransposon RNA. Nat Commun $2014 ; 5: 5276$.

40. Randow F, Youle RJ. Self and nonself: how autophagy targets mitochondria and bacteria. Cell Host Microbe 2014 ; 15 : 403-11.

41. Salemi S, Yousefi S, Constantinescu MA, et al. Autophagy is required for selfrenewal and differentiation of adult human stem cells. Cell Res $2012 ; 22$ : $432-5$. 


\section{RÉFÉRENCES}

42. Shaw AC, Goldstein DR, Montgomery RR. Age-dependent dysregulation of innate immunity. Nat Rev Immunol $2013 ; 13: 875-87$.

43. Zoncu R, Efeyan A, Sabatini DM. mTOR: from growth signal integration to cancer, diabetes and ageing. Nat Rev Mol Cell Biol $2011 ; 12: 21-35$.

44. Senovilla L, Galluzzi L, Zitvogel L, Kroemer G. Immunosurveillance as a regulator of tissue homeostasis. Trends Immunol $2013 ; 34: 471-81$.

45. Kroemer G, Senovilla L, Galluzzi L, et al. Natural and therapy-induced immunosurveillance in breast cancer. Nat Med $2015 ; 21: 1128-38$.

46. Zhong Z, Umemura A, Sanchez-Lopez $\varepsilon$, et al. NF-kappaB Restricts Inflammasome Activation via Elimination of Damaged Mitochondria. Cell 2016 ; $164: 896-910$.

47. He C, Bassik MC, Moresi V, et al. Exercise-induced BCL2-regulated autophagy is required for muscle glucose homeostasis. Nature $2012 ; 481: 511-5$.

48. Saitoh T, Fujita N, Hayashi T, et al. Atg9a controls dsDNA-driven dynamic translocation of STING and the innate immune response. Proc Natl Acad Sci USA 2009 ; 106 : 20842-6.

49. Krysko DV, Garg AD, Kaczmarek A, et al. Immunogenic cell death and DAMPs in cancer therapy. Nat Rev Cancer $2012 ; 12: 860-75$
50. Ko A, Kanehisa A, Martins I, et al. Autophagy inhibition radiosensitizes in vitro, yet reduces radioresponses in vivo due to deficient immunogenic signalling. Cell Death Differ $2014 ; 21$ : 92-9.

51. Madeo F, Pietrocola F, Eisenberg T, Kroemer G. Caloric restriction mimetics: towards a molecular definition. Nat Rev Drug Discov $2014 ; 13$ : 727-40.

52. Rubinsztein DC, Codogno $P$, Levine $B$. Autophagy modulation as a potential therapeutic target for diverse diseases. Nat Rev Drug Discov 2012 ; 11 : 709 30.

53. Vigié $P$, Camougrand N. Mitophagie et contrôle qualité des mitochondries. Med Sci (Paris) 2017 ; $33: 231-7$.
TIRÉS À PART

L. Galluzzi

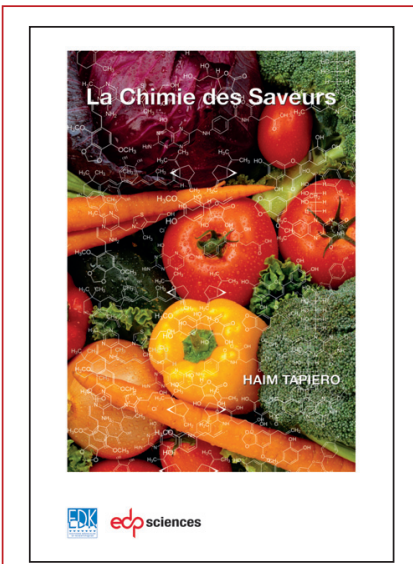

a cuisine est une science. II existe une relation étroite entre élaborer une recette et entreprendre une recherche scientifique. Quelle que soit l'origine d'une recette, d'un livre ou inventée, il faudra faire le choix des ingrédients, les mélanger et les cuire de manière appropriée afin de ne pas altérer les substances actives qui composent les ingrédients.

Une fois la cuisson terminée, il faudra analyser le goût et si nécessaire prévoir son amélioration. Améliorer une recette nécessite de connaître le ou les processus qui interviennent dans le développement des arômes, des saveurs et de la texture. Cette approche est similaire à celle développée par le scientifique.

La relation entre l'élaboration des recettes, les substances nutritives qui composent les ingrédients et la santé de l'homme est issue de plusieurs disciplines de la recherche fondamentale et clinique. Au cours des dernières années, de nombreux travaux scientifiques ont été publiés sur le rôle de la nutrition et la réduction des risques dans les pathologies comme les maladies cardio-vasculaires ou les cancers.

Le but principal de cet ouvrage a été d'identifier la structure chimique des composants actifs des ingrédients utilisés en cuisine (légumes, herbes aromatiques, épices) et qui entrent dans la préparation des recettes pour « végétariens » et « omnivores ».

ISBN : 978-2-7598-1137-3 180 pages

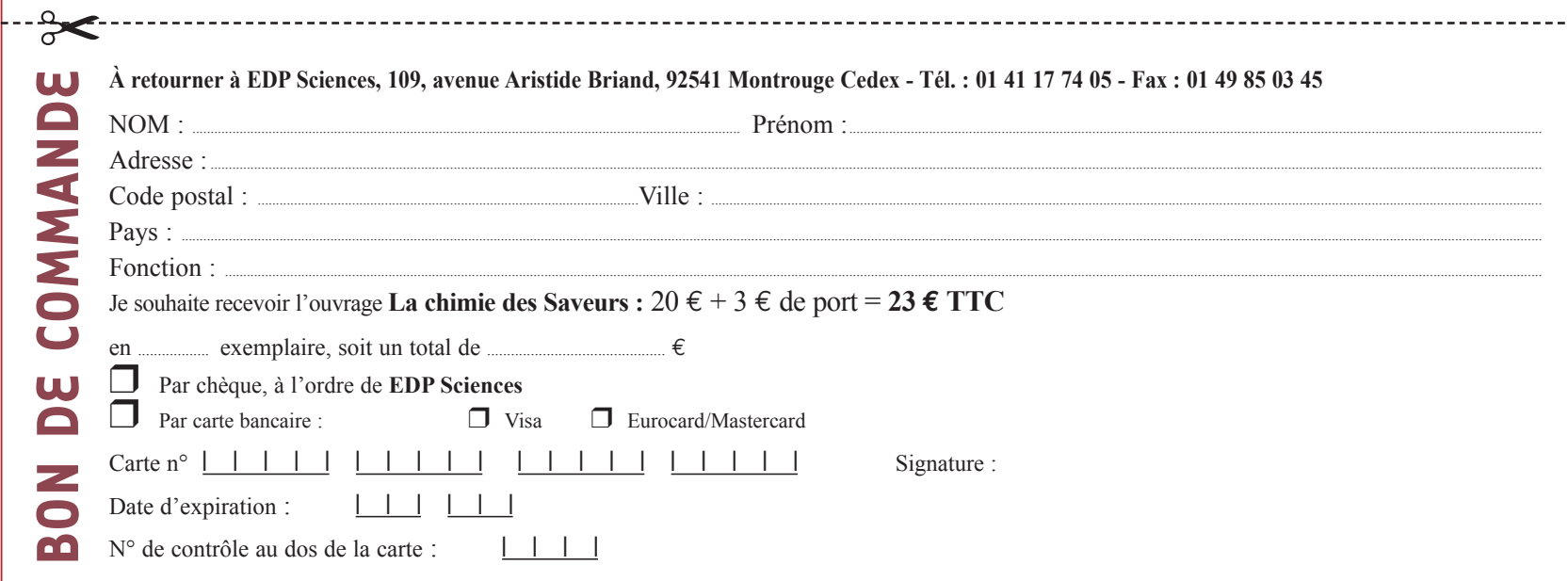

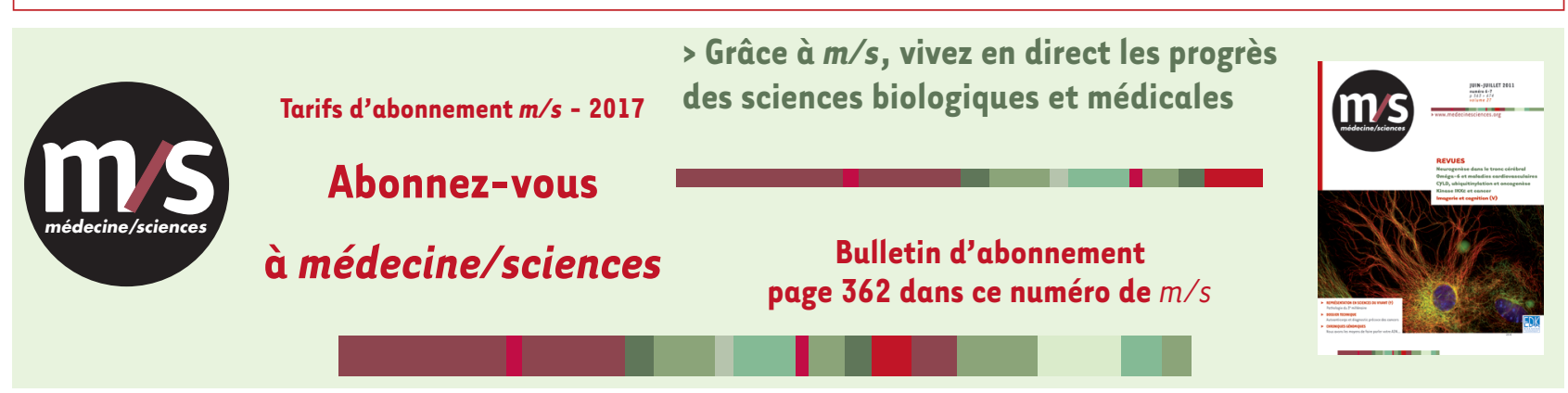

